\title{
Further insights into the proton spin with the new HERMES Recoil Detector
}

\author{
I. Vilardi \\ (on behalf of the HERMES collaboration) \\ University of Bari, Department of Physics, Via Orabona 4, 70126 Bari, Italy \\ Ignazio.Vilardi@ba.infn.it
}

\begin{abstract}
The HERMES experiment, installed in the $27.5 \mathrm{GeV}$ HERA lepton ring at DESY/Hamburg, is used to study the spin structure of the nucleon. To get information about the orbital angular momentum $L_{q}$ of quarks, exclusive DIS reactions are investigated. The HERMES Collaboration installed a new Recoil Detector to upgrade the existing spectrometer to improve the study of hard exclusive processes, detecting recoil protons with low momentum. Deeply Virtual Compton Scattering is the main process to be studied. The HERMES Recoil Detector consists of three subcomponents inside a superconducting magnet that provides a longitudinal superconducting magnetic field of 1 Tesla. The Recoil Detector was installed in January 2006 and commissioning started in February. First results from the detector will be presented.
\end{abstract}

Keywords: Generalized Parton Distributions, Deeply Virtual Compton Scattering, recoil proton PACS: $01.30 . \mathrm{Cc}, 14.20 . \mathrm{Dh}, 29.30 . \mathrm{Aj}, 29.40 . \mathrm{Gx}, 29.40 . \mathrm{Mc}, 29.40 . \mathrm{Vj}, 29.40 . \mathrm{Wk}$

\section{INTRODUCTION}

Generalized Parton Distributions (GPD's) offer a way to unify the previously disjunct pictures of the nucleon given by Parton Distribution Functions on the one hand and nucleon Form Factors on the other. For the first time GPD's may provide detailed information on the localization of partons inside hadrons and access to their orbital angular momentum $L_{q}$ [1]. GPD's can be accessed experimentally through the measurement of hard exclusive reactions, the cleanest of which [2] is the Deeply Virtual Compton Scattering (DVCS) (Figure 1, left panel). In the reaction $e p \rightarrow e^{\prime} \gamma p$ at the beam energy of HERMES (27.5 GeV), the Bethe-Heitler process (Figure 1, right panel) dominates over DVCS in most of the kinematic region. However, measurable asymmetries in beam spin and beam charge arise from the interference of both processes. The beam spin asymmetry is proportional to the imaginary part of the DVCS amplitude, while the beam charge asymmetry is proportional to the real part of the DVCS amplitude. Both can in turn be expressed in terms of GPD's.

\section{THE RECOIL DETECTOR}

The HERMES Recoil Detector [3] has been designed to upgrade the HERMES spectrometer [4] for the measurement of hard exclusive reactions, in particular DVCS. Its objectives are the detection and identification of recoil protons and the rejection of background events, coming mainly from intermediate $\Delta$-production and from semi-inclusive 


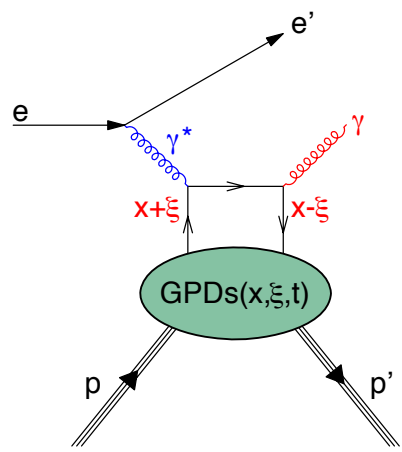

DVCS

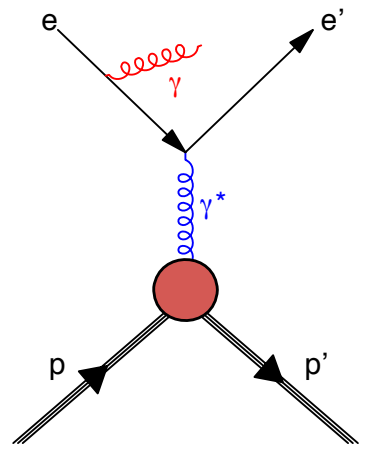

Bethe-Heitler

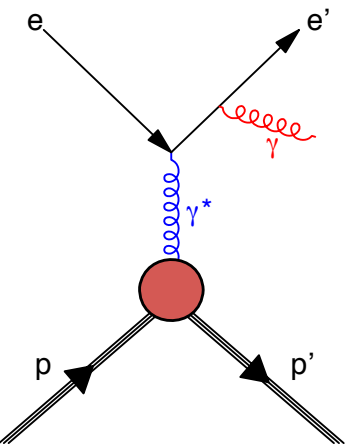

FIGURE 1. Feynman graphs of DVCS (left panel) and Bethe-Heitler (BH) processes (right panel). Both processes lead to the same final state, thus their amplitudes interfere.

processes. The Recoil Detector is shown in Figure 2: it consists of three active components: a Silicon Strip Detector surrounding the target cell inside the beam vacuum, a Scintillating Fibre Tracker and a Photon Detector consisting of three layers of tungsten/scintillator. All three detectors are located inside a superconducting solenoidal magnetic field of 1 Tesla. Together these detectors cover most of the kinematic region for DVCS/Bethe-Heitler events. The technical details of the different subsystems of the Recoil Detector will be described in the next subsections.

\section{The Silicon Detector}

The closest sub-detector to the beam axis is the Silicon Strip Detector (SSD). The SSD is located inside the scattering chamber within the beam vacuum in order to detect recoil protons with momentum in the range of $135-400 \mathrm{MeV} / c$. momentum and particle type of the detected particle are determined from the energy deposited in the silicon layers. Due to the very low energy of the recoil protons it is necessary to minimize the amount of material between the interaction point and the detector. Therefore the SSD is mounted inside the HERA ring vacuum in a scattering chamber. For this reason, all of the components and techniques used for the SSD must be vacuum compatible. The SSD consists of 8 modules mounted in two layers symmetrically around the target cell in roof-shaped structures. The main components of the SSD include two TIGRE (The Tracking and Imaging Gamma Ray Experiment) sensors and two readout hybrids with digital control and analog readout circuits. The double-sided silicon TIGRE sensors are from Micron Semiconductors Ltd with a size of $9.9 \times 9.9 \mathrm{~cm}^{2}$. The active thickness of the sensors is $300 \mu \mathrm{m}$. On each side of the sensor there are 128 strips with a 758 $\mu \mathrm{m}$ wide pitch. The necessary momentum dynamic range is established by splitting the signal into a high gain and a low gain path: each strip is read out by one HELIX chip connected directly to the sensor and by a second chip connected via a $10 \mathrm{pF}$ capacitor [5]. The total surface area of the 16 silicon sensors constituting the SSD is $0.16 \mathrm{~m}^{2}$, 


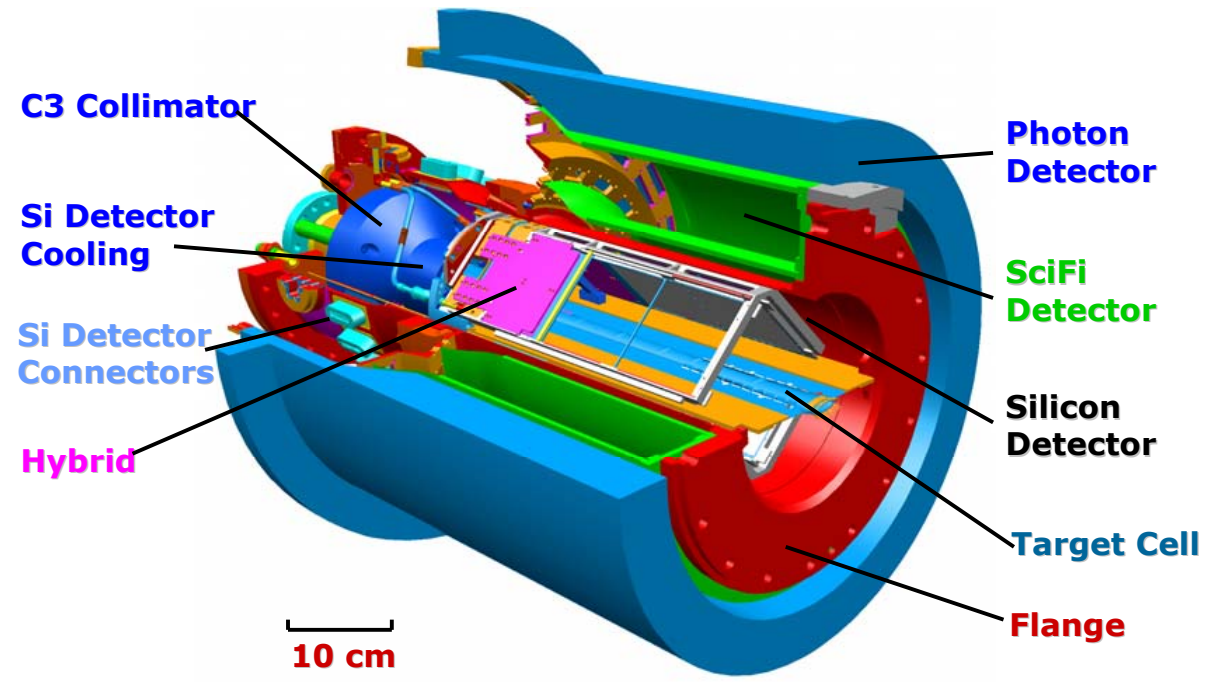

FIGURE 2. Schematic drawing of the Recoil Detector and its support structure. The electron beam enters from the left side, where an additional collimator protects the Recoil Detector from synchrotron radiation.

resulting in 4096 strips. The strip directions of the p-side and the n-side in one sensor are arranged perpendicularly to each other so that 2-dimensional position information is available.

\section{The Scintillating Fibre Tracker}

The Scintillating Fibre Tracker (SFT) is the second detector located after the SSD, going from inside. Protons of higher momenta (above $250 \mathrm{MeV} / c$ ) escape the 1.2 thick Aluminium scattering chamber are detected by the SFT. Their momentum is determined by the deflection of their track in the longitudinal magnetic field which also allows the identification of the sign of the charge.

The SFT consists of two concentric barrels of scintillating fibres. Kuraray SCSF-78M with a diameter of $1 \mathrm{~mm}$ were chosen as active material for the SFT. Each SFT barrel in turn consists of two sub-barrels, resulting in 5120 channels in total, but, whereas the inner sub-barrel is oriented parallel to the beam axis, the outer sub-barrel is inclined by $10^{\circ}$ in a stereo configuration. This configuration allows the determination of a space point of a particle track for each barrel. The scintillating fibres are connected via $4 \mathrm{~m}$ long light guides made of clear fibres to Multi-Anode Photo-Multiplier Tubes. 


\section{The Photo Detector}

The outermost sub-detector of the Recoil Detector is the Photon Detector (PD). The PD improves the capability of the Recoil Detector to suppress background by rejecting semi-inclusive events in which a $\pi^{0}$ is produced and events in which an intermediate $\Delta$-resonance is produced. This is achieved by detecting at least one of the photons into which a neutral pion emitted from a $\Delta$-decay subsequently decays. Also, the first layer of the PD improves the pion/proton separation capability of the SFT: a pion rejection factor of ten is expected for momenta up to $800 \mathrm{MeV} / c$ [3]. Finally, the PD provides a method by which to align the subcomponents of the Recoil Detector by acting as a trigger for cosmic ray events. The PD is constructed from six layers, alternating between a tungsten converter layer from which incident charged particles produce electromagnetic showers, and a scintillator layer which detects these showers. The inner layer is segmented into 60 trapezoidal blocks aligned parallel to the beam axis, the middle and outer layers are segmented into 44 blocks, aligned at +45 and -45 degrees to the beam.

\section{The Magnet}

The Recoil Detector is finally surrounded by a Superconducting (SC) 1 Tesla solenoid magnet. The primary purpose of the Recoil Detector SC magnet is to provide a means for the SFT to measure track momentum. Additionally, the magnet protects the SSD from background electrons emitted from Møller scattering events by allowing these electrons to spiral forward in the magnetic field.

\section{FIRST DATA}

The Recoil Detector was installed in January 2006 and started to take data in February. The tracking in the magnetic field using the SFT was possible with the Recoil Detector SC magnet. In March the target cell was damaged while it was being inspected which resulted in large radiation doses to the SSD once the beam was injected. This required rebuilding the SSD in March - June. End of June HERA switched the beam polarity from negative to positive. The data taking was continued with the fully installed Recoil Detector in July 2006 till the $30^{\text {th }}$ of June 2007, last day of HERA running.

As a demonstration of the performance of the SSD, Figure 3 shows the energy loss in the inner layer of the SSD versus the energy loss in the outer silicon layer. The black line in the Figure represents a Monte Carlo prediction. The upper line represents protons which punch through the first layer but get stuck in the second and the lower line represents protons which punch through both layers. Figure 4 shows the energy deposits in individual detection layers (SSD on the left panel and SFT on the right panel): from the Figure a well defined $\mathrm{p} / \pi^{+}$separation can be easily seen for both detectors $\left(\pi^{-}\right.$are recognized from their charge). Figure 5 shows the energy deposits in the SSD (same quantities shown in Figure 4 on the left side) after a cut on the missing mass $\left(M_{X}<1.7\right.$ $\mathrm{GeV}$ ), evaluated from the spectrometer: comparing it to the plot shown in Figure 4, it 


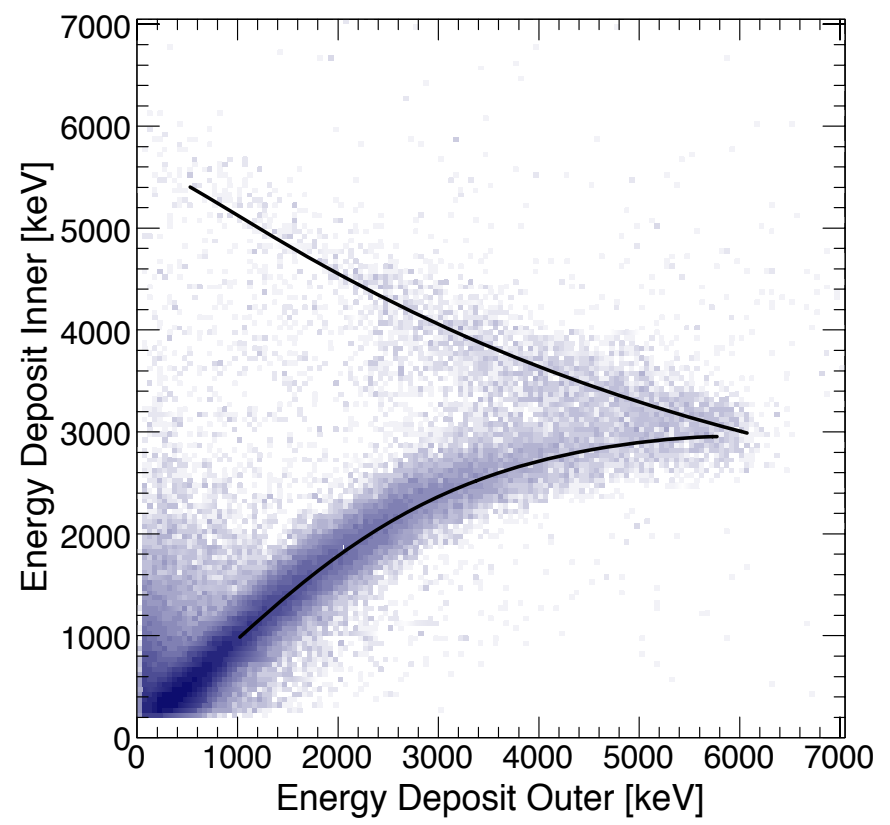

FIGURE 3. Energy loss in the inner silicon layer versus the energy loss in the outer silicon layer. The black line represents a Monte Carlo prediction.
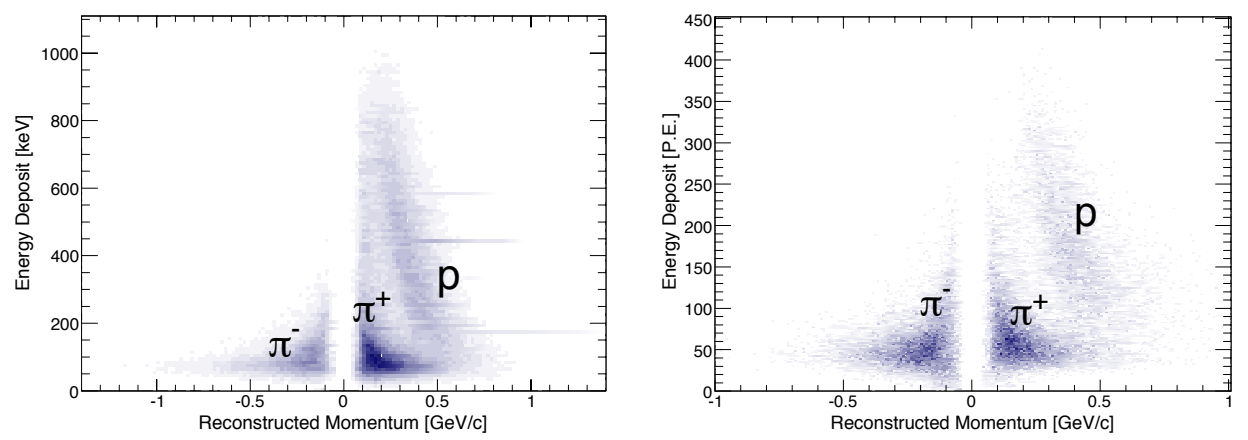

FIGURE 4. Energy deposits in individual detection layers (SSD on the left and SFT on right).

can be easily seen that only protons survive to this cut, as well as a small amount of $\pi^{+}$ coming from the $\Delta^{+}$-decay.

\section{CONCLUSIONS}

The HERMES Collaboration installed in January 2006 a new Recoil Detector to upgrade the existing spectrometer. The Recoil Detector will improve the study of hard exclusive 


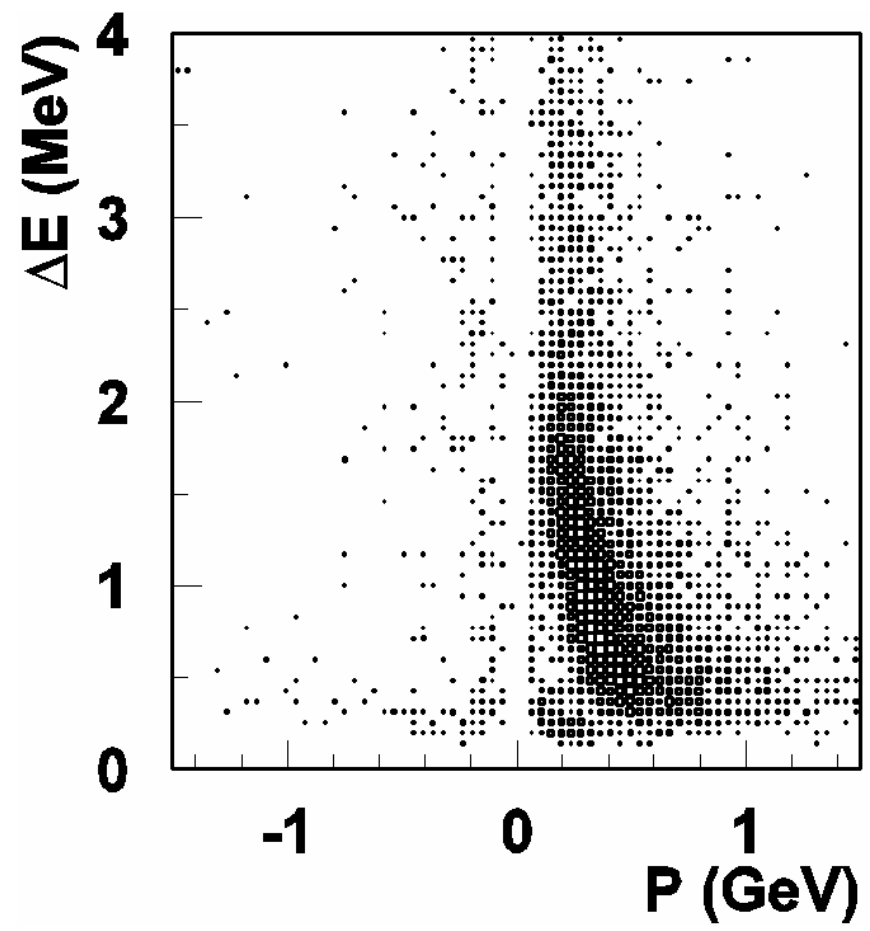

FIGURE 5. Energy deposits in the SSD (same quantities shown in Figure 4 on the left side) after a missing mass cut $\left(M_{X}<1.7 \mathrm{GeV}\right)$.

processes, detecting recoil protons with low momentum, coming from the DVCS and Bethe-Heitler processes. Since September '06, the Recoil Detector was fully operational and it ran stably for 10 months without any problems. 28 millions of DIS events from hydrogen target and 7 millions DIS events from deuterium target were collected. As seen in Figure 4 the Recoil Detector is able to provide particle identification, through energy deposition in the different detector layers, to positively identify the recoil proton. Moreover the Recoil Detector can also be used as a tool to measure background contribution in the previous Hermes DVCS analysis, done only with the spectrometer.

\section{REFERENCES}

1. X.-D. Ji, Phys. Rev. Lett., 78, 610-613, 1997.

2. X.-D. Ji, Phys. Rev., D55, 7114-7125, 1997.

3. B. Seitz, Nucl. Instrum. and Meth., A535, 538-541 2004.

4. K. Ackerstaff et al. (HERMES Collaboration), Nucl. Instrum. and Meth., A417, 230-265, 1998.

5. M. Reinecke et al., IEEE Trans. Nucl. Sci., 51, 1111-1116, 2004. 